\title{
On formality of homogeneous Sasakian manifolds
}

https://doi.org/10.1515/coma-2019-0009

Received January 21, 2019; accepted April 16, 2019

Abstract: In this note we show families of homogeneous Sasakian manifolds $G / H$ which are nonformal. The non-formality condition is expressed in terms of characters of a maximal torus in $G$.

\section{Introduction}

It is well known that the existence of a geometric structure on a manifold may impose topological restrictions on it. One of such restrictions is formality. This property holds for Kaehler manifolds. In this paper we consider the odd-dimensional counterpart of this class, that is, Sasakian manifolds. Let $(M, \eta)$ be a co-oriented contact manifold with a contact form $\eta \in \Omega^{1}(M)$, that is $\eta \wedge(d \eta)^{n}>0$ everywhere, with $\operatorname{dim} M=2 n+1$. We say that $(M, \eta)$ is $K$-contact if there is an endomorphism $\Phi$ of $T M$ such that:

- $\Phi^{2}=-\operatorname{Id}+\xi \otimes \eta$, where $\xi$ is the Reeb vector field of $\eta$ (that is $i_{\xi} \eta=1, i_{\xi}(d \eta)=0$ ),

- the contact form $\eta$ is compatible with $\Phi$ in the sense that $d \eta(\Phi X, \Phi Y)=d \eta(X, Y)$, for all vector fields $X, Y$,

- $d \eta(\Phi X, X)>0$ for all nonzero $X \in \operatorname{ker} \eta$, and

- the Reeb field $\xi$ is Killing with respect to the Riemannian metric defined by the formula $g(X, Y)=$ $d \eta(\Phi X, Y)+\eta(X) \eta(Y)$.

In other words, the endomorphism $\Phi$ defines a complex structure on $\mathcal{D}=$ ker $\eta$ compatible with $d \eta$, hence $\Phi$ is orthogonal with respect to the metric $\left.g\right|_{\mathcal{D}}$. By definition, the Reeb vector field $\xi$ is orthogonal to ker $\eta$, and it is a Killing vector field.

Let $(M, \eta, g, \Phi)$ be a K-contact manifold. Consider the contact cone as the Riemannian manifold $C(M)=$ $\left(M \times \mathbb{R}^{>0}, t^{2} g+d t^{2}\right)$. One defines the almost complex structure $I$ on $C(M)$ by:

- $I(X)=\Phi(X)$ on ker $\eta$,

- $I(\xi)=t \frac{\partial}{\partial t}, I\left(t \frac{\partial}{\partial t}\right)=-\xi$, for the Killing vector field $\xi$ of $\eta$.

We say that $(M, \eta, \Phi, g, I)$ is Sasakian if $I$ is integrable. Thus, by definition, any Sasakian manifold is Kcontact.

There is much interest in constructing K-contact manifolds which do not admit Sasakian structures. The odd Betti numbers up to dimension $n$ of Sasakian $(2 n+1)$-manifolds must be even. The parity of $b_{1}$ was used to produce the first examples of K-contact manifolds with no Sasakian structure [5, example 7.4.16]. More refined tools are needed in the case of even Betti numbers. The cohomology algebra of a Sasakian manifold satisfies a version of a hard Lefschetz property [6]. Using it examples of K-contact non-Sasakian manifolds are produced in [7] in dimensions 5 and 7. These examples are nilmanifolds with even Betti numbers, so in particular they are not simply connected. When one moves to the case of simply connected manifolds, Kcontact non-Sasakian examples of any dimension $\geq 9$ were constructed in [10] using the evenness of the third

Irena Morocka-Tralle: Department of Mathematics and Computer Science University of Warmia and Mazury Słoneczna 54, 10-710 Olsztyn, Poland, E-mail: itralle@matman.uwm.edu.pl

*Corresponding Author: Aleksy Tralle: Department of Mathematics and Computer Science University of Warmia and Mazury Słoneczna 54, 10-710 Olsztyn, Poland, E-mail: aleksymath46@gmail.com 
Betti number of a compact Sasakian manifold. Simply connected examples in dimension 7 were constructed in [11]. In [3] it is proved that all higher order Massey products for simply connected Sasakian manifolds vanish, although there are Sasakian manifolds with non-vanishing triple Massey products. This yields examples of simply connected K-contact non-Sasakian manifolds in dimensions $\geq 17$. Thus, in general Sasakian manifolds are not formal. Various examples of both, formal and non-formal Sasakian manifolds do exist [3], however, it is not easy to decide whether the given Sasakian manifold is formal or not. In order to understand this fact better we want to look at the simplest case of compact homogeneous Sasakian manifolds. The aim of this note is to characterize the formality of compact homogeneous Sasakian manifolds in terms of the representation theory. By a theorem which can be derived from a result of Alekseevsky [1] one can describe all homogeneous Sasakian manifolds $G / H$ in terms of a functional $\theta \in \mathfrak{g}^{\star}$. If $\theta$ is a weight of the maximal torus $T \subset G$ (see Section 3) we are able to prove the (non) formality results in terms of $\theta$. Assume that $G / H$ is a compact homogeneous Sasakian manifold, and the maximal torus of $H$ has the form $T_{\theta} \subset H$, where $\theta$ is a weight, that is, a differential of a character $\lambda \in X(T)$ of the maximal torus $T$. Here $T_{\theta}$ is a subtorus whose Lie subalgebra has the form $\mathfrak{t}_{\theta}=\operatorname{Ker} \theta$. It can be shown that a weight $\theta$ can be extended onto $\mathfrak{g}$, and, therefore, $\theta \in \mathfrak{g}^{*}$ (see [12], p. 219). We will say that the homogeneous Sasakian manifold $G / H$ is determined by the weight $\theta$ (see Definition 3). Note that generically, Sasakian manifolds are of this type (see Section 3). We prove the following.

Theorem 1. Let $G / H$ be a compact homogeneous Sasakian manifold determined by a dominant weight $\theta$. Assume that $G$ is a classical Lie group of rank $G=l>7$. Assume that $\theta$ has numerical labels $\lambda_{i}$ satisfying the condition $\lambda_{i}>0$ for at least one $i$ such that $3 \leq i<l-3$, with respect to the fixed ordering in the root system of $\mathfrak{g}$. Then $G / L_{\theta}$ is non-formal. If $G$ is an exceptional Lie group of types $E_{6}, E_{7}$ and $E_{8}$, then for any $\theta$ whose numerical labels satisfy $\lambda_{i}>0$ for $2 \leq i \leq l-1$, the Sasakian manifold $G / L_{\theta}$ is non-formal.

It should be noted that M. Amann [2] developed several general methods of constructing non-formal compact homogeneous spaces (see Section 6). Some families of non-formal homogeneous spaces from this work are actually Sasakian. However, we want to obtain an answer directly in terms of the data of the given homogeneous Sasakian manifold. Finally, let us mention the recent paper [9] devoted to formality of homogeneous 3-Sasakian manifolds. It would be interesting to develop an approach to the problems considered in [9] along the lines of this paper.

\section{Preliminaries}

\subsection{Boothby-Wang fibrations}

Let $(N, \omega)$ be a compact symplectic manifold with an integral symplectic form $\omega$. Consider the principal circle bundle

$$
S^{1} \longrightarrow M \stackrel{\pi}{\longrightarrow} N
$$

whose Euler class is $[\omega] \in H^{2}(N, \mathbb{Z})$. Then the total space $M$ admits a contact form $\eta$ such that $\pi^{\star}[\omega]=d \eta$. The converse also holds: if $(M, \eta)$ is a contact manifold with a free circle action preserving the contact form, then the base of the principal circle bundle (1) is symplectic and $\pi^{\star}[\omega]=d \eta$. This construction is called the Boothby-Wang fibration (see [4]). In the metric case of K-contact or Sasakian manifolds one can say more (see [5], Chapter 7). We restrict ourselves to the Sasakian case. A Sasakian structure $(\eta, \Phi, g, I)$ on a manifold $M$ is called quasi-regular if there is a positive integer $\delta$ satisfying the condition that each point of $M$ has a foliated chart $(U, t)$ with respect to the Reeb field $\xi$ (the coordinate $t$ is in the direction of $\xi$ ) such that each leaf for $\xi$ passes though $U$ at most $\delta$ times. If $\delta=1$, then the Sasakian structure is called regular. Not all Sasakian structures are regular, but any manifold which admits a Sasakian structure, admits a quasi-regular one [5] (Theorem 7.1.10 in the K-contact case, the same is valid for Sasakian structures). Regular Sasakian structures can be characterized via the Boothby-Wang fibrations as follows. 
Theorem 2. A total space of a Boothby-Wang fibration (1) over a compact Kaehler manifold with an integral Kaehler form $[\omega]$ admits a regular Sasakian structure with underlying contact form $\eta$ that satisfies the equation $d \eta=\pi^{\star} \omega$. Conversely, if $M$ is a regular Sasakian manifold,then there is a free circle action on $M$ determining the structure of a Boothby-Wang fibration over a Kaehler manifold $N$ with an integral Kaehler form $[\omega]$. The Euler class of this fibration is $[\omega]$.

\subsection{Lie groups, characters and weights}

We freely use the basics of Lie theory without explanations, the reader can consult [13]. Lie algebras corresponding to Lie groups $G, H, \ldots$ are denoted by the corresponding Gothic letters $\mathfrak{g}, \mathfrak{h}, \ldots .$. A character of a Lie group is its 1-dimensional representation. Let $T$ be a maximal torus in a compact Lie group $G$, and let $X(T)$ be the group of characters of $T$. Any character $\lambda \in X(T)$ defines a linear mapping $d \lambda: \mathfrak{t} \rightarrow i \mathbb{R}$. Clearly $d \lambda$ can be extended to a complex linear form on $\mathfrak{t}^{c}$. Hence $d \lambda \in(i t)^{\star}$. Clearly, the correspondence $\lambda \rightarrow d \lambda$ is a monomorphism $X(T) \hookrightarrow(i t)^{\star}$. Let $\Delta$ be a root system of $\mathfrak{g}^{c}$ with respect to $\mathfrak{t}^{c}$. The additive subgroup $Q \subset(i t)^{\star}$ generated by $\Delta$ is a lattice in $(i t)^{*}$ of rank $l=\operatorname{rank} G$. Any set $\Pi$ of simple roots is a basis of $Q$. Let $\Delta^{\vee}$ be the dual root system with the set of simple roots $\Pi^{\vee}=\left\{\alpha_{1}^{\vee}, \ldots, \alpha_{l}^{\vee}\right\}$. Then $\xi_{i}$ determined by the equations $\xi_{i}\left(\alpha_{j}^{\vee}\right)=\delta_{i j}, i=1, \ldots, l, j=1, \ldots, l$ is called the fundamental weight. Let $P$ be a lattice generated by the fundamental weights. For any $\lambda \in P$ one defines the integers $\lambda_{i}=\lambda\left(\alpha_{i}^{\vee}\right)$. Thus, $\lambda_{i}$ are the coordinates of $\lambda$ relative to the basis consisting of the fundamental weights. The numbers $\lambda_{i}$ are called the numerical labels of $\lambda$. We call $\lambda$ dominant, if $\lambda_{i} \geq 0$ for all $i$.

\subsection{Formality}

In this subsection some definitions and results about minimal models and Massey products are reviewed [8].

We work with differential graded commutative algebras, or DGAs, over the field $\mathbb{R}$ of real numbers. The degree of an element $a$ of a DGA is denoted by $|a|$. A DGA $(\mathcal{A}, d)$ is minimal if:

i) $\mathcal{A}$ is free as an algebra, that is, $\mathcal{A}$ is the free algebra $\wedge V$ over a graded vector space $V=\bigoplus_{i} V^{i}$, and

ii) there is a collection of generators $\left\{a_{\tau}\right\}_{\tau \in I}$ indexed by some well ordered set $I$, such that $\left|a_{\mu}\right| \leq\left|a_{\tau}\right|$ if

$\mu<\tau$ and each $d a_{\tau}$ is expressed in terms of preceding $a_{\mu}, \mu<\tau$. This implies that $d a_{\tau}$ does not have a linear part.

In our context, the main example of DGA is the de Rham complex $\left(\Omega^{\star}(M), d\right)$ of a differentiable manifold $M$, where $d$ is the exterior differential.

Given a differential graded commutative algebra $(\mathcal{A}, d)$, we denote its cohomology by $H^{\star}(\mathcal{A})$. The cohomology of a differential graded algebra $H^{\star}(\mathcal{A})$ is naturally a DGA with the product structure inherited from that on $\mathcal{A}$ and with the differential being identically zero. The DGA $(\mathcal{A}, d)$ is connected if $H^{0}(\mathcal{A})=\mathbb{R}$, and $\mathcal{A}$ is 1-connected if, in addition, $H^{1}(\mathcal{A})=0$. Morphisms between DGAs are required to preserve the degree and to commute with the differential.

We shall say that $(\bigwedge V, d)$ is a minimal model of the differential graded commutative algebra $(\mathcal{A}, d)$ if $(\bigwedge V, d)$ is minimal and there exists a morphism of differential graded algebras

$$
\rho:(\bigwedge V, d) \longrightarrow(\mathcal{A}, d)
$$

inducing an isomorphism $\rho^{\star}: H^{\star}(\bigwedge V) \stackrel{\sim}{\longrightarrow} H^{\star}(\mathcal{A})$ of cohomologies.

We say that two DGAs $\left(\mathcal{A}, d_{A}\right)$ and $\left(\mathcal{B}, d_{B}\right)$ are quasi-isomorphic, if there is a sequence of DGA algebras $\left(\mathcal{A}_{i}, d_{i}\right)$ and a sequence of morphisms between $\left(\mathcal{A}_{i}, d_{i}\right)$ and $\left(\mathcal{A}_{i+1}, d_{i+1}\right)$ with $\left(\mathcal{A}_{1}, d_{1}\right)=\left(\mathcal{A}, d_{A}\right)$ and $\left(\mathcal{A}_{n}, d_{n}\right)=$ $\left(\mathcal{B}, d_{B}\right)$ such that these morphisms induce isomorphisms of the corresponding cohomology algebras (the morphisms may be directed arbitrarily).

Any connected differential graded algebra $(\mathcal{A}, d)$ has a minimal model unique up to isomorphism [8].

A minimal model of a connected differentiable manifold $M$ is a minimal model $(\bigwedge V, d)$ for the de Rham complex $\left(\Omega^{\star}(M), d\right)$ of differential forms on $M$. If $M$ is a simply connected manifold, then the dual of the real 
homotopy vector space $\pi_{i}(M) \otimes \mathbb{R}$ is isomorphic to $V^{i}$ for any $i$. This relation also holds when $i>1$ and $M$ is nilpotent, that is, the fundamental group $\pi_{1}(M)$ is nilpotent and its action on $\pi_{j}(M)$ is nilpotent for all $j>1$.

Recall that a minimal algebra $(\bigwedge V, d)$ is called formal if there exists a morphism of differential algebras $\psi:(\bigwedge V, d) \longrightarrow\left(H^{*}(\bigwedge V), 0\right)$ inducing the identity map on cohomology. Also a differentiable manifold $M$ is called formal if its minimal model is formal. Many examples of formal manifolds are known: spheres, projective spaces, compact Lie groups, some homogeneous spaces, flag manifolds, and all compact Kähler manifolds $[8,14,15]$.

Finally, let us mention that quasi-isomorphic minimal algebras have isomorphic minimal models. Therefore, to study formality of manifolds, one can use other "algebraic models", that is, DGAs $\left(\mathcal{A}, d_{A}\right)$ which are quasi-isomorphic to the de Rham algebra, and which may be not minimal. This will be used in our study of (non) formality of homogeneous spaces.

\subsection{Formality of compact homogeneous spaces}

In this subsection we recall some methods of establishing the formality property in the case of compact homogeneous spaces $G / H$ of compact semisimple Lie groups. In this case the minimal model of $G / H$ is also the minimal model of a free differential graded algebra associated with $G / H$. This algebra is called the Cartan algebra. Also, in many cases one can associate with a Cartan algebra some numerical invariants which yield some necessary conditions for formality. These numerical invariants will be the basic tool in this paper. The basic reference is [12]. Let $G$ be a compact connected Lie group. The cohomology algebra $H^{\star}(G)$ is an exterior algebra over vector space $P_{G}$ generated by primitive elements $y_{1}, \ldots, y_{n}$ of odd degrees $2 k_{1}-1, \ldots, 2 k_{n}-1$, where $n=\operatorname{rank} G$. These generators can be chosen in a way, that the cohomology algebra of the classifying space $B G$ is isomorphic to the polynomial algebra $\mathbb{R}\left[x_{1}, \ldots, x_{n}\right]$, where elements $y_{j}$ are cohomology classes in the universal space $E G$ containing coboundaries of the transgression for $x_{j}$. Let $H \subset G$ be a closed subgroup. Choose maximal tori $T_{H} \subset T$ of $H$ and $G$, and their Lie algebras $\mathfrak{t}_{H} \subset \mathfrak{t}$. It is well known that the cohomology algebras $H^{\star}(B G)$ and $H^{\star}(B H)$ are isomorphic to the polynomial algebras of the invariants of the Weyl groups $W(G)$ and $W(H)$ respectively. Denote these rings as $\mathbb{R}[\mathfrak{t}]^{W(G)}$ and $\mathbb{R}\left[\mathfrak{t}_{H}\right]^{W(H)}$. It is a classical result that the canonical homomorphism $\rho^{\star}(H, G): H^{\star}(B G) \rightarrow H^{\star}(B H)$ induced by the inclusion $H \hookrightarrow G$ sends any invariant polynomial from $H^{\star}(B G)$ to its restriction on $H^{\star}(B H)$. Introduce the following notation:

$$
\begin{gathered}
H^{\star}(B G) \cong \mathbb{R}[\mathfrak{t}]^{W(G)}=\mathbb{R}\left[f_{1}, \ldots, f_{n}\right], \\
H^{\star}(B H) \cong \mathbb{R}\left[\mathfrak{t}_{H}\right]^{W(H)}=\mathbb{R}\left[u_{1}, \ldots, u_{r}\right],
\end{gathered}
$$

where $f_{j}$ are $W(G)$-invariant polynomials over $\mathfrak{t}$, and $u_{k}$ are $W(H)$-invariant polynomials over $\mathfrak{t}_{H}$, generating the rings of invariants $\mathbb{R}[\mathfrak{t}]^{W(G)}$ and $\mathbb{R}\left[\mathfrak{t}_{H}\right]^{W(H)}$, respectively. By $j^{\star}: \mathbb{R}[\mathfrak{t}]^{W(G)} \rightarrow \mathbb{R}\left[\mathfrak{t}_{H}\right]^{W(H)}$ we denote the restriction map $j^{*}(f)=\left.f\right|_{t_{H}}$. The differential graded algebra

$$
\begin{aligned}
\left(C_{G / H}, d\right) & =\left(H^{\star}(B H) \otimes \Lambda\left(y_{1}, \ldots, y_{n}\right), d\right) \\
\left.d\right|_{H^{*}(B H)} & =0, d y_{j}=j^{\star}\left(f_{j}\right), j=1, \ldots, n
\end{aligned}
$$

is called the Cartan algebra of $G / H$. It is a classical result that $\left(C_{G / H}, d\right)$ is quasi-isomorphic to the de Rham algebra $\Omega^{\star}(G / H)$, and, therefore, can serve as an algebraic model of $G / H$. The minimal model of $\left(C_{G / H}, d\right)$ is isomorphic to the minimal model of $G / H$. Therefore, in case of compact homogeneous spaces one may use it to study the formality property. Let us describe the main numerical invariant which we use in this article. For any polynomial ideal $I \subset \mathbb{K}\left[x_{1}, \ldots, x_{m}\right]$ of a graded polynomial algebra over a field $\mathbb{K}$ define an algebraic affine variety $V(I)$ over the algebraic closure of $\mathbb{K}$. Let $b_{1}, \ldots, b_{r}$ be the minimal system of homogeneous generators of $I$. By definition, the deficiency $\mathrm{df}(I)$ is a number

$$
\operatorname{df}(I)=\operatorname{dim} \bar{I}-m=r-m,
$$


where $\bar{I}=I / B_{+} I, B=\mathbb{K}\left[x_{1}, \ldots, x_{m}\right]$. Now, consider the Cartan algebra $\left(C_{G / H}, d\right)$. Assume that $f_{1}, \ldots, f_{n}$ are generators of the ring $\mathbb{R}[\mathfrak{t}]^{W(G)}$, and assume that $r=\operatorname{rank} H$. The deficiency of $G / H$ is defined as

$$
\operatorname{df}(G / H)=\operatorname{df}\left(j^{*}\left(f_{1}\right), \ldots, j^{*}\left(f_{n}\right)\right) .
$$

This is the homotopy invariant of $G / H$ (see [12], p.210).

Theorem 3 ([12], Theorem 2, p. 211). Let G/H be a homogeneous space of a compact Lie group G.The following two conditions are equivalent.

i) $\mathrm{G} / \mathrm{H}$ is formal;

ii) $\operatorname{df}(G / H)=0$.

Theorem 4 ([12], p.212). The homogeneous space $G / H$ is formal if and only if the homogeneous space $G / T_{H}$ is formal.

Remark 1. A more general formulation and proof can be found in [2], Proposition D.

Now we are ready to formulate a result which yields numerical invariants detecting non-formality. Throughout this work $P(M, t)$ denotes the Poincare polynomial of a manifold $M$.

Theorem 5 ([12], Proposition 3, p.220). Let $M=G / T_{\chi}$ be a homogeneous space associated with a non-trivial character of a maximal torus $T$ of a compact simple Lie group $G$, and $K \supset T_{X}$ be a subgroup whose Lie algebra has the form of a centralizer $\mathfrak{k}=\mathfrak{z}_{\mathfrak{g}}(z)$ for some $z \in \mathfrak{t}$. Suppose that $P_{G}$ and $P_{K}$ are graded by degrees $2 p_{i}-1$ and $2 q_{i}-1, i=1, \ldots, l=\operatorname{rank} G=l$. Write down the polynomial

$$
p_{a}(M, t)=\left(1-t^{2}\right) \prod_{i=1}^{l} \frac{\left(1-t^{2 p_{i}}\right)}{\left(1-t^{2 q_{i}}\right)}
$$

in the fom

$$
p_{a}(M, t)=q_{0}(M, t)-q_{1}(M, t)
$$

where $q_{0}$ and $q_{1}$ are the sums of the terms with positive and negative coefficients, respectively. Then

$$
p(M, t)=q_{0}+\frac{1}{t} q_{1}(t)
$$

Also, $b_{2 s}(M)=0$ for $2 s>v=\frac{1}{2} \operatorname{dim} G / K$, and $b_{2 s-1}(M)=0$ for $2 s-1<v=\frac{1}{2} \operatorname{dim} G / K$.

Corollary 1 ([12], p. 221). If $G$ is simple, $M=G / T_{\chi}$ and $\mathrm{df}(M)=0$, then

$$
v \leq \frac{2 \operatorname{dim} G}{\operatorname{rank} G}-3 .
$$

\section{Co-oriented homogeneous contact manifolds}

Let $\mathfrak{g}$ be a Lie algebra, and $\theta \in \mathfrak{g}^{\star}$. Consider the coadjoint orbit $\mathcal{O}_{\theta}=G / G_{\theta} \subset \mathfrak{g}^{\star}$. Here $G_{\theta}$ denotes the isotropy subgroup of $\theta$. We will denote by $\mathfrak{g}_{\theta}$ the Lie subalgebra of $G_{\theta}$.

Theorem 6 (Alekseevsky, [1]). Let $G$ be a compact Lie group and let $\mathfrak{l}_{\theta} \subset \mathfrak{g}_{\theta}$ be the Lie subalgebra defined by

$$
\mathfrak{l}_{\theta}=\left\{X \in \mathfrak{g}_{\theta} \mid \theta(X)=0, X \in \mathfrak{g}_{\theta}\right\} .
$$

Assume that $\mathfrak{l}_{\theta}$ has codimension 1 in $\mathfrak{g}_{\theta}$ and that the Lie subgroup $L_{\theta} \subset G_{\theta}$ corresponding to $\mathfrak{l}_{\theta}$ is closed. Then $G / L_{\theta}$ admits an invariant contact form, and the fiber bundle 


$$
G_{\theta} / L_{\theta} \rightarrow G / L_{\theta} \rightarrow G / G_{\theta}
$$

is the Boothby-Wang fibration over the coadjoint orbit $G / G_{\theta}$. Any homogeneous co-oriented contact manifold is obtained in this way.

Note that there is no proof in [1], therefore, we formulate the result in more detail and give some explanations.

Theorem 7. If $G$ is a compact connected Lie group and $L \subset G$ is a closed subgroup, then $G / L$ admits a $G$ invariant contact form $\eta$ if and only if the following holds:

i) $L=L_{\theta}$ is a codimension 1 closed subgroup of the isotropy subgroup $G_{\theta}$ of $\theta \in g^{\star}$ under the coadjoint action, where $\theta \in \mathfrak{g}^{\star}$ is determined by the $G$-invariant contact form $\eta$;

ii) the group $G_{\theta}$ is a semidirect product $L_{\theta} \ltimes S^{1}$, thus $L_{\theta}$ is a normal subgroup of $G_{\theta}$ and the projection $G_{\theta} \rightarrow$ $G_{\theta} / L_{\theta} \cong S^{1}$ is an $L_{\theta}$-principal fibration;

iii) $\eta$ yields an $S^{1}$-invariant connection on the principal circle fibration $p: G / L_{\theta} \rightarrow G / G_{\theta}$;

iv) $d \eta$ is equal, up to a constant, to $p^{\star} \omega$, where $\omega$ is the G-invariant symplectic form defined by the coadjoint action on the coadjoint orbit $G / G_{\theta}$;

v) the Euler class of the fibration $p: G / L_{\theta} \rightarrow G / G_{\theta}$ is equal, up to a non-zero constant, to the cohomology class of $\omega$.

vi) the subgroup $G_{\theta}$ is equal to the centralizer of a circle subgroup in $G$.

Proof. Let us denote by $\mathfrak{g}, \mathfrak{g}^{\star}$ the Lie algebra and the dual Lie algebra of $G$ respectively. The form $\eta$ gives on $G$ an invariant 1-form, thus an element $\theta \in \mathfrak{g}^{\star}$. Consider the vector $V \in \mathfrak{g}$ dual to $\theta$ (where duality is given by the Killing form of $G$ ). Then $V$ generates a torus $S$ in $G$ and $G_{\theta}$ is the centralizer of $S$. The latter is equivalent to the assumption (i). The subgroup $L_{\theta}$ is generated by the kernel of $\theta$. If the closure of a 1-parameter subgroup in a torus is of dimension greater than 1 , then the element in $\mathfrak{g}^{\star}$ dual to the subgroup must have at least one irrational coefficient in an integral base of the Lie algebra $\mathfrak{s}$ of the torus $S$. This implies that the kernel of $\theta$ is not closed if $\operatorname{dim} S>1$, thus we see that under our assumptions $S$ is 1-dimensional.

The Lie subalgebra of the subgroup $L_{\theta}$ of $G_{\theta}$ is generated by the ideal Ker $\theta$ hence it is a normal subgroup of $G_{\theta}$. We get therefore an $L_{\theta}$-principal fibration $L_{\theta} \rightarrow G / L_{\theta} \rightarrow G / L_{\theta}$. The vector field $V^{\star}$ on $G / L_{\theta}$ defines an $S^{1}-$ action and $\theta\left(V^{\star}\right)=$ const $=c>0$.

The symplectic form on a coadjoint orbit of $\theta$ is defined by the formula $\omega(X, Y)=\theta([X, Y])=d \eta(X, Y)$. Thus, up to a constant $d \eta=p^{\star} \omega$. The property ( $v$ ) follows directly from (iv), the property ( $\left.v i\right)$ is clear.

Theorem 8. Any compact simply connected homogeneous Sasakian manifold of a semisimple compact Lie group $G$ has the form $G / L_{\theta}$ for an element $\theta \in \mathfrak{g}^{\star}$. Conversely, if $L_{\theta}$ is closed than $G / L_{\theta}$ is a homogeneous Sasakian manifold.

Proof. Any compact simply connected homogeneous Sasakian manifold $G / H$ is a co-orineted contact manifold with a $G$-invariant contact structure. Therefore, one applies Theorem 7 to get $H=L_{\theta}$ and a BoothbyWang fibration $G / L_{\theta} \rightarrow G / G_{\theta}$. Conversely, for $G / L_{\theta}$ one considers the corresponding Boothby-Wang fibration $G / L_{\theta} \rightarrow G / G_{\theta}$. By Theorem 7 one can assume that the Euler class of this fibration is $[\omega]$. Moreover, the coadjoint orbit $G / G_{\theta}$ is Kaehler (this is always true for homogeneous symplectic manifolds of compact semisimple Lie groups [15], p.142). Therefore, $G / L_{\theta}$ is Sasakian, and the underlying contact structure $\eta$ is $G$-invariant and is determined by $\theta \in \mathfrak{g}^{\star}$. The $G$-invariance of $\eta$ follows from [5], Theorem 8.3.6. It follows from the definition and the invariance of the integrable complex structure $I$ in $G / G_{\theta}$ that the Sasakian structure $(\eta, \Phi, g, I)$ must be $G$-invariant as well. 


\section{Centralizers of tori}

\subsection{Centralizers in semisimple complex Lie algebras}

In this (and only this) section we assume that $\mathfrak{g}$ is a complex Lie algebra, and $\mathfrak{t}$ is a (fixed) Cartan subalgebra.

Definition 1. A subalgebra $\mathfrak{f} \subset \mathfrak{g}$ is said to be regular with respect to $\mathfrak{t}$, if $\mathfrak{t} \subset \mathfrak{n}_{\mathfrak{g}}(\mathfrak{f})$.

Definition 2. A subsystem $\Gamma \subset \Delta$ is called closed, if

$$
\alpha, \beta \in \Gamma \text { and } \alpha+\beta \in \Delta \Rightarrow \alpha+\beta \in \Gamma \text {. }
$$

The subsystem $\Gamma \subset \Delta$ is called symmetric if $-\Gamma=\Gamma$.

Proposition 1 ([13]). Let $\mathfrak{g}$ be a semisimple Lie algebra., and $\mathfrak{t}$ is a Cartan subalgebra. Then all regular (with respect to $\mathfrak{t}$ ) subalgebras $\mathfrak{f} \subset \mathfrak{g}$ can be written in the form

$$
\mathfrak{f}=\mathfrak{f}(\mathfrak{t}, \Gamma)=\mathfrak{t}_{1}+\sum_{\alpha \in \Gamma} \mathfrak{g}_{\alpha},
$$

where $\Gamma$ is a closed subsystem in $\Delta, \mathfrak{t}_{1} \subset \mathfrak{t}$ for all $\alpha \in \Gamma \cap(-\Gamma)$.

Proposition 2 ([13]). Assume that $\mathfrak{g}$ is semisimple, and $\Gamma_{1}$ and $\Gamma_{2}$ be closed subsystems in $\Delta$. Assume that $\mathfrak{f}\left(\mathfrak{t}, \Gamma_{1}\right)$ and $\mathfrak{f}\left(\mathfrak{t}, \Gamma_{2}\right)$ are subalgebras of maximal rank. Then

$$
\Gamma_{1} \subset \Gamma_{2} \Longleftrightarrow \mathfrak{f}\left(\mathfrak{t}, \Gamma_{1}\right) \subset \mathfrak{f}\left(\mathfrak{t}, \Gamma_{2}\right) .
$$

For any subsystem $R \subset \Delta$ we denote by $[R]$ the set of all roots in $\Delta$ that are integral linear combinations of of roots in $R$. Any closed symmetric subsystem $\Gamma \subset \Delta$ is a root system of the subalgebra $f(t, \Gamma)$ and, therefore, can be represented as $\Gamma=[R]$, where $R$ is the system of simple roots in $\Gamma$. A subsystem $R \subset \Delta$ is called a $\pi$-system, if $R$ is linearly independent and $\alpha-\beta \notin \Delta$ for all $\alpha, \beta \in R$.

Proposition 3 ([13]). Any $\pi$-system $R \subset \Delta$ is a system of simple roots of the symmetric closed subsystem $\Gamma=[R]$. Conversely, any system of simple roots of a symmetric closed subsystem is a $\pi$-system in $\Delta$.

Theorem 9 ([13]). Suppose that a reduced root system $\Delta$ is indecomposable and $R \subset \Delta$ is a $\pi$-system. A system $\Gamma=[R]$ is a maximal proper closed symmetric subsystem of $\Delta$ if and only if $R$ can be obtained from the extended system of simple roots $\tilde{\Pi}=\left\{\alpha_{0}, \alpha_{1}, \ldots, \alpha_{l}\right\}$ in one of the following ways:

i) $R=\tilde{\Pi} \backslash\left\{\alpha_{i}\right\}, n_{i}$ is a prime number, $\operatorname{rank} \Gamma=l$,

ii) $R=\Pi \backslash\left\{\alpha_{i}\right\}, i>0, n_{i}=1, \operatorname{rank} \Gamma=l-1$.

Theorem 10 ([13]). Let $R \subset \Pi$, where $\Pi$ is a set of simple roots of $\Delta$. Then the subalgebra $\mathfrak{f}(\mathfrak{t},[R])$ is of the form $\mathfrak{z} \mathfrak{g}(C)$ for some $C \subset \mathfrak{g}$. Conversely, any subalgebra $\mathfrak{z} \mathfrak{g}(C), C \subset \mathfrak{t}$ is of the form $\mathfrak{f}(\mathfrak{t},[R])$, where $R$ is a subset of a set of simple roots $\Pi \subset \Delta$.

Corollary 2. Let $R=\Pi \backslash\left\{\alpha_{i}\right\}$. For any $R^{\prime} \subset R$ the following inclusion holds:

$$
\mathfrak{f}\left(\mathfrak{t},\left[R^{\prime}\right]\right) \subset \mathfrak{f}(\mathfrak{t},[R]) .
$$

Proof. The proof follows from Propositions 2, 3 and Theorems 9 and 10.

Let $\theta$ be a weight. Consider the subset

$$
R=\{\alpha \in \Pi \mid(\theta, \alpha)=0\}
$$

Then for the Lie algebra $f(t,[R])$ there is a connected Lie subgroup $K=H_{R}$, which is a centralizer of a torus in $G$ (see [12], p. 65). 


\section{Non-formal homogeneous Sasakian manifolds}

\subsection{General position Sasakian manifolds}

Assume that $G$ is a compact semisimple Lie group, and $T$ is a maximal torus in $G$ with the group of characters $X(T)$. Choose a non-trivial character $\lambda \in X(T)$. Let $\theta \in \mathfrak{t}^{\star}$ be the weight determined by $\lambda$. Extend it to $\theta \in \mathfrak{g}^{\star}$ and consider the coadjoint orbit $\mathcal{O}_{\theta}=G / G_{\theta}$. One can see that $G_{\theta} \supset T$, and the maximal torus in $G_{\theta}$ has the form $T_{\theta}\left(\mathfrak{t}_{\theta}=\operatorname{Ker} \theta\right)$. Then one can see that $L_{\theta} \subset G_{\theta}$ has the maximal torus $(\operatorname{Ker} \theta)^{0}$. We get the contact manifold $G / L_{\theta}$. From these observations one derives that therefore, $G / G_{\theta} \subset \mathfrak{g}^{*}$ is a coadjoint orbit. By the same reason, $G / L_{\theta}$ is a homogeneous contact manifold determining the Boothby-Wang fibration

$$
G_{\theta} / L_{\theta} \rightarrow G / L_{\theta} \rightarrow G / G_{\theta}
$$

As a result, we get the following.

Definition 3. We will say that a contact manifold $G / L_{\theta}$ is determined by a character of a maximal torus.

Theorem 11 ([12], p. 220). Let $G / L_{\theta}$ be compact homogeneous Sasakian manifold of a compact semisimple Lie group $G$ of rank $>3$ determined by a character of a maximal torus. If the corresponding weight $\theta$ has the property $(\theta, \alpha) \neq 0$ for any $\alpha \in \Delta$, then $G / L_{\theta} i$ is non-formal.

Proof. We need to show that $d f\left(G / L_{\theta}\right)=0$. By Theorem 4 it is sufficient to show that $\operatorname{df}\left(G / T_{\theta}\right)=0$. Apply Corollary 1 and get the inequality $(n-l) / 2 \leq 2 n / l-3$, because in this case $R=\emptyset$, and $K=H_{R}=T$, where $T$ is a maximal torus in $G$. One can see that this inequality may hold only if $l \geq 3$.

Remark 2. If $T_{\theta}=\operatorname{Ker} \theta$ with $(\theta, \alpha) \neq 0$, it is said that $T_{\theta}$ is in general position. Thus, general position subtori of codimension 1 always yield non-formal Sasakian manifolds.

\subsection{Proof of Theorem 1}

One can see that Theorem 3 implies that one needs to check if $\operatorname{df}\left(G / L_{\theta}\right)=0$. If this equality is valid, then necessarily Corollary 1 holds. Thus, the proof goes as folows: one considers each of the types of $G$, that is,

$$
A_{l}, B_{l}, C_{l}, D_{l}, E_{6}, E_{7}, E_{8}
$$

separately. In each case, one considers the fundamental weight $\xi_{i}, i=1, \ldots, l$ and the system of simple roots $\Pi=\left\{\alpha_{1}, \ldots, \alpha_{l}\right\}$. Then for the fundamental weights $\xi_{i}$ we get $\left(\xi_{i}, \alpha_{j}\right)=\delta_{i j}$, which shows that for any fundamental weight, say, $\xi_{p}$

$$
R \subset \Pi, R=\left\{\alpha_{j} \in \Pi \mid\left(\xi_{p}, \alpha_{j}\right)=0\right\}=\left\{\alpha_{1}, \ldots \hat{\alpha_{p}}, \ldots, \alpha_{l}\right\}
$$

Thus, for $\xi_{p}, T_{\xi_{p}}=\operatorname{Ker} \xi_{p}$ determines a Sasakian manifold $G / L_{\xi_{p}}$. Following [12] we define $K=H_{R}$ for each type of $G$. For example, if $G$ has type $A_{l}$, we get $\mathfrak{k}=\mathfrak{s u}(p-1)+\mathfrak{s u}(l-p)+\mathbb{R}$. Therefore,

$$
\operatorname{dim} K=(p-1)(p+1)+(l-p)(l-p+2)+1 .
$$

In each case we get $\operatorname{dim} K$ expressed via $p$ and $l$. Substituting these numbers to the inequality for $v=$ $\frac{1}{2} \operatorname{dim} G / K$ in Corollary 2, we get the result. Note that in each case the inequalities are slightly different. We write down the result of the calculation and indicate when the inequality in Corollary 1 is not satisfied.

i) type $A_{l}: 3 \leq p \leq l-3, l>7$;

ii) type $B_{l}: 3 \leq p \leq l-3$, and $l \geq 5$;

iii) type $C_{l}, 3 \leq p \leq l-3, l \geq 5$;

iv) type $D_{l}: 3 \leq p \leq l-3, l>7$; 
v) $E_{6}, E_{7}, E_{8}, 2 \leq l-1, l=6,7,8$.

The formulation of Theorem 1 unifies the results above. Now, we complete the proof by noticing that if the weight $\theta$ is dominant and satisfies the restrictions of Theorem 1, the inequalities above will be also true, because of Corollary 2.

\section{Other examples}

In some cases one can derive the non-formality results from the following theorem.

Theorem 12 (Amann, [2]). Let $E^{2 n+1}$ be a simply connected space with finite dimensional rational cohomology and let $B^{2 n}$ be a simply connected space satisfying the Hard Lefschetz property (for $n \geq 1$ ). Suppose there is a fibration $S^{1} \rightarrow E \rightarrow B$. Assume that the following holds true:

- The Euler class of $p$ is a non-vanishing multiply of the Kahler class of B in rational cohomology.

- The rational cohomology of $B$ is concentrated in even degrees only.

- The rational homotopy groups of $B$ are concentrated in degrees smaller or equal to $n$.

Then $E$ is a non-formal space.

Clearly, the Boothby-Wang fibration $S^{1} \rightarrow G / L_{\xi} \rightarrow G / G_{\xi}$ satisfies the first two conditions of Theorem 12, but not necessarily the third one. For example, in order to apply this theorem, one needs to demand that the top rational homotopy of $B=G / G_{\xi}$ does not lie above the half of its dimension. In particular, in case $G=S p(n)$ (note that now we use Amann's notation) one considers non-formal homogeneous spaces of the form

$$
S p(n) / S U(p) \times S p(l), p+l=n .
$$

Then, the third condition in Theorem 12 implies

$$
\frac{1}{2} p^{2}+\left(2 l-\frac{7}{2} p\right)-4 l+1 \geq 1,
$$

which is not necessarily satisfied under the assumptions of Theorem 1.

Here are examples of formal compact homogeneous Sasakian manifolds.

Example 1 ([12], Examples 3 and 4 on p. 221). The spaces $S p(l) / S U(l)$ and $S O(2 l+1) / S U(l)$ have zero deficiency for $l \leq 4$, and, therefore, are formal.

\section{References}

[1] D. Alekseevsky, Contact homogeneous spaces, Functional Anal. Appl. 24(1990), no. 4, 74-75

[2] M. Amann, Non-formal homogeneous spaces, Math. Z. 274(2012), 1299-1325

[3] I. Biswas, M. Fernández, V. Muñoz, A. Tralle, On formality of Sasakian manifolds, J. Topol. 9(2016), 161-180

[4] D. Blair, Riemannian Geometry and Contact Manifolds, Springer, Berlin, 1978.

[5] C. Boyer, K. Galicki, Sasakian Geometry, Oxford, 2007

[6] B. Cappelletti-Montano, A. de Nicola, I. Yudin, Hard Lefschetz theorem for Sasakian manifolds, J. Different. Geom. 101(2015), 47-66.

[7] B. Cappelletti-Montano, A. de Nicola, J. C. Marrero, I. Yudin, Examples of compact K-contact manifolds with no Sasakian metric, Int. J. Geom. Methods Mod. Physics 11(2014), 1460028

[8] Y. Félix, S. Halperin, J.-C. Thomas, Rational Homotopy Theory, Springer, Berlin, 2002

[9] M. Fernández, V. Muñoz, J. Sánchez, On SO(3)-bundles over the Wolf spaces, ArXiv: 1709.08806

[10] B. Hajduk, A. Tralle, On simply connected K-contact non-Sasakian manifolds, J. Fixed Point Theory Appl. 16(2014), 229-241

[11] V. Muñoz, A. Tralle, Simply connected K-contact and Sasakian manifolds of dimension 7, Math. Z. 281(2015), 457-470

[12] A.L. Onishchik, Topology of transitive transformation groups, Johann Ambrosius Barth, Leipzig, 1993

[13] A. L. Onishchik, E. B. Vinberg, Lie Groups and Lie Algebras III, Springer, Berlin, 1994 
[14] Z. Stępień, On formality of a class of compact homogeneous spaces, Geom. Dedicata 93(2002), 37-45

[15] A. Tralle, J. Oprea, Symplectic Manifolds with no Kaehler structure, Springer, Berlin, 1997 